\title{
Personality and Body Weight
}

\section{-Mechanisms, Longitudinal Associations and Context}

\author{
Angelina R. Sutin \\ Florida State University College of Medicine
}

\author{
Antonio Terracciano \\ Florida State University College of Medicine
}

Models of personality and health have grown in complexity as more is discovered about how traits are related to health-related behaviors, morbidity, and mortality. The present article applies a model of personality and health that incorporates longitudinal relations, behavioral and physiological mechanisms, and context to the relation between Five-Factor Model personality traits and body mass index (BMI) and obesity (BMI $\geq 30$ ). Conscientiousness is associated consistently with lower body weight; the relation between the other traits and BMI is more complex. Conscientiousness is also associated with risk of obesity over time, and specific aspects of Conscientiousness and Neuroticism are associated with greater weight gain and are also sensitive to changes in weight over time. Behavioral (e.g., physical activity) and physiological (e.g., inflammation) factors explain part of the association between personality and BMI. Finally, the broader social environment shapes the expression of personality in relation to body weight. This review highlights replicable associations between personality and BMI and potential mechanisms of this association. Future research needs to better address how specific aspects of the social and family environment moderate the relation between personality and BMI and take a lifespan perspective to better incorporate how traits contribute to weight starting in childhood.

Key words: Five-Factor Model, body mass index, obesity, personality, lifespan models

As defined by the Five Factor Model (FFM; McCrae \& Costa, 2003), the characteristic ways that people think, feel, and behave are operationalized along five broad domains: Neuroticism, Extraversion, Openness, Agreeableness, and Conscientiousness. These traits have been shown to be stable over time (Roberts \& DelVecchio, 2000), observable by others (McCrae et al., 2004), and universal (McCrae et al., 2005). Decades of longitudinal research have pointed to the relevance of these personality traits to important life outcomes across a range of domains. Within the psychological domain, for example, personality predicts depression (Kendler \& Myers, 2010); within the academic domain, personality predicts academic achievement (Noftle \& Robins, 2007); within the occupational domain, personality predicts objective job performance (Barrick \& Mount, 1991); and within the health domain, personality predicts morbidity (Sutin, Zonderman, Ferrucci, \& Terracciano, 2013) and mortality (Jokela, Batty, et al., 2013).

The association between personality and longevity in particular ignited a great interest in understanding how a trait measured in childhood could go on to predict how long someone would 
live six decades later (Friedman et al., 1993). This interest led to numerous replications that indicate that higher Conscientiousness is associated with living a longer life (Chapman, Fiscella, Kawachi, \& Duberstein, 2010; Jokela, Batty, et al., 2013; Kern \& Friedman, 2008; Terracciano, Löckenhoff, Zonderman, Ferrucci, \& Costa, 2008). This robust association between Conscientiousness and longevity further led to identifying behavioral (Turiano, Chapman, Gruenewald, \& Mroczek, 2015) and physiological (Hampson, Edmonds, Goldberg, Dubanoski, \& Hillier, 2013) mechanisms that explain this association. In tandem with this growing evidence base, there has been a shift in focus toward intermediate conditions or diseases that are potential targets of intervention and are of relevance to the health and quality of the individual's life. This research has further expanded on the potential impact of health conditions on personality development. We first review briefly models of personality and health before turning more specifically to the relation between personality traits and both body mass index (BMI) and obesity (BMI $\geq 30)$.

\section{Models of Personality and Health}

Theoretical models of FFM personality traits and health have been developed to explain how personality contributes to health outcomes. As the evidence grows, these models have increased in complexity. The most common models of personality and health used today are so-called "second generation" models in which behavioral, psychological, physiological, and social factors are hypothesized to mediate the relation between personality traits and a health outcome, such as longevity (Friedman, Kern, Hampson, \& Duckworth, 2014). Third-generation models are more complex and incorporate several additional components. For example, these models recognize a biological basis of both personality and longevity, that the relation between personality and mechanisms of health play out across the lifespan, and that there are dynamic relations between personality and social processes that contribute to the individual's health.

The present paper reviews the evidence for how personality traits are associated with BMI and obesity. This review of the literature is structured around a model of personality and health that includes mediators, time, and the social environment. Specifically, personality is expected to be associated with BMI/obesity (direct effect) through both behavioral and physiological mechanisms (indirect/mediated effect). These processes should play out over time (longitudinal association), be bidirectional (reciprocal relation), and may be sensitive to the environment in which the individual moves (moderated by context).

\section{Personality and Body Mass Index}

In the United States, the rate of obesity has more than doubled since 1980 (Ogden, Yanovski, Carroll, \& Flegal, 2007). The United States is not alone in this increase. Although there is substantial variation in how much the rates have changed across the globe, most countries have seen at least some increase over the past few decades (Finucane et al., 2011). This increase in the prevalence of obesity, coupled with an increase in the potential health risks associated with obesity (e.g., type 2 diabetes), has led to research into the etiology of weight gain. It is recognized that the development of obesity is multifaceted with risk factors that range across the continuum from genetics to public policy (Gortmaker et al., 2011). Within this 
range, a number of psychological factors, including personality, are thought to play a role. As with any individual risk factor, the association might be modest in size, but replicable relations indicate a significant role of personality in body weight. To summarize what is known about the relation between personality and BMI, we apply a model of personality and health to this relation and describe the evidence for each pathway in the model.

Cross-sectional associations. The first step in applying a model of personality and health is to establish an association between the traits and the health outcome of interest (i.e., BMI/obesity). Of the five traits, Conscientiousness is the trait most consistently associated with BMI and obesity. Across multiple populations and different questionnaire measures of personality, individuals who score higher in Conscientiousness tend to have lower BMI and are less likely to be obese (Chapman, Fiscella, Duberstein, Coletta, \& Kawachi, 2009; Magee \& Heaven, 2011; Mõttus et al., 2013; Sutin \& Terracciano, 2016b; Terracciano et al., 2009). This association tends to be similar across age groups, but there is some evidence that it is moderated by sex: Conscientiousness is protective for both sexes, but the negative relation tends to be stronger for women (Brummett et al., 2006; Sutin \& Terracciano, 2016a).

The relation between Neuroticism and body weight is not as straightforward. There is some evidence that a greater tendency to experience negative emotions and stress is associated with higher BMI and risk of obesity (Armon, Melamed, Shirom, Shapira, \& Berliner, 2013; Sutin, Ferrucci, Zonderman, \& Terracciano, 2011). Not all studies, however, find this relation (Chapman et al., 2009; Mõttus et al., 2013). Several factors may contribute to this complexity. First, the relation might be moderated by sex; the association tends to be stronger among women than among men (Sutin \& Terracciano, 2016a). Similar moderation is found for constructs conceptually related to Neuroticism, such as depressive symptoms (Sutin \& Zonderman, 2012). Second, Neuroticism increases risk for eating disorders (Ferguson, Muñoz, \& Winegard, 2012), and there may be a nonlinear association between this trait and BMI. That is, individuals with either underweight or obesity may be higher in Neuroticism, which would obscure the linear relation. Finally, differences in the content of personality measures may contribute to differences in associations seen across studies. Scales with items that tap in to the impulsivity and anger facets of Neuroticism tend to show more consistent associations with BMI (Sutin et al., 2011) than scales with items that tap into the emotional vulnerability aspects of Neuroticism (Jokela, Hintsanen, et al., 2013).

The relation between Extraversion and body weight is likewise complicated. Similar to Neuroticism, there is some evidence that the relation between Extraversion and BMI varies by sex. That is, some studies find that men who score higher in Extraversion have a higher BMI, whereas women do not show this association (Brummett et al., 2006; Sutin \& Terracciano, 2016a). Not all studies, however, find this association (Terracciano et al., 2009). As a main effect, Extraversion is sometimes associated with higher BMI (Armon et al., 2013; Sutin et al., 2011) and is sometimes unrelated to BMI (Magee \& Heaven, 2011; Mõttus et al., 2013). The association between both Agreeableness and Openness and BMI is likewise ambiguous (e.g., Armon et al., 2013; Brummett et al., 2006; Chapman et al., 2009; Magee \& Heaven, 2011). 
Longitudinal associations. Personality is not just associated with BMI when measured concurrently, but it also predicts change in weight and risk of developing obesity over time. A meta-analysis of personality traits and risk of obesity found that individuals who scored higher on Conscientiousness were at lower risk of obesity across the follow-up period, an association found in six independent cohorts (Jokela, Hintsanen, et al., 2013). In addition, specific aspects of Conscientiousness have been found to predict healthier weight gain over time. Individuals who score higher in self-discipline and order tend to gain less weight across adulthood (Sutin et al., 2011). Although Neuroticism tends to be unrelated to obesity risk over time (Jokela, Hintsanen, et al., 2013), specific aspects of this trait are associated with greater weight gain (Sutin et al., 2011). Early in adulthood, for example, individuals who score higher in impulsivity, a facet of Neuroticism, weigh, on average, about 15 pounds more than individuals who score lower on impulsivity; by older adulthood, this difference increases to about 34 pounds (Sutin et al., 2011).

In addition to weight gain, personality is also associated with variability in weight over time. Although weight tends to increase across adulthood until a slight decline in old age, there is tremendous variability in the rate of weight gain and in fluctuations over time. That is, some people cycle through periods of weight loss and regain, some increase steadily across adulthood, and others remain relatively stable. Personality is associated with this variability. Specifically, individuals who score higher in the trait tendency toward negative emotionality fluctuate in weight more across adulthood than those who score lower in this tendency, whereas individuals who score higher in the tendency to be responsible and disciplined tend to fluctuate less than those who score lower in this tendency (Sutin et al., 2011).

Reciprocal relations. The association between personality and body weight is unlikely to be unidirectional; personality and body weight are likely to shape each other over time. Longitudinal studies with multiple assessments of both personality and BMI allow the opportunity to examine whether there are bidirectional associations between personality and body weight. When baseline BMI or obesity is used as a predictor of personality development, it tends to be unrelated to change in the traits: Whether someone measures in the obese weight category or not is unrelated to change in personality over time (Jokela, Hintsanen, et al., 2013).

There are other ways to conceptualize how weight might be related to personality development. Significant weight gain, for example, has physiological, psychological, and social effects on the individual; these associations extend to specific aspects of personality. Individuals who gain at least $10 \%$ of their body weight over time, for example, increase in both their tendency to give in to their urges (i.e., increases in Impulsivity) and their tendency to think before acting (i.e., increases in Deliberation) (Sutin, Costa, et al., 2013). The increase in impulsivity associated with weight gain was expected; individuals who gain weight come to see themselves as more impulsive perhaps because they observe their impulsive behaviors that contributed to the weight gain. The relation between weight gain and increases in Deliberation, however, was surprising. This pattern suggests that individuals are not unaware of their weight gain; they may not be eating mindlessly, but they may lack the emotional control to 
be able to stop themselves.

Behaviors conducive to obesity. Personality is not just associated with BMI and risk of obesity but is also associated with a number of behaviors that are conducive to obesity. These factors may be one behavioral mechanism that links personality to risk of weight gain over time. Specifically, personality has been associated with physical activity, sedentary behavior, and eating-related behavior, and these behaviors, in turn, increase risk of obesity.

Several meta-analyses have summarized the literature on personality and physical activity (Rhodes \& Smith, 2006; Sutin et al., 2016; Wilson \& Dishman, 2015). Extraversion tends to be the trait that is most consistently associated with physical activity: Individuals who are more sociable and outgoing tend to exercise more frequently than individuals who score lower on this trait. Both Neuroticism and Conscientiousness are likewise associated with physical activity: Individuals who are prone to feeling negative emotions and vulnerability to stress exercise less, whereas individuals who are more responsible and disciplined tend to exercise more. Finally, Openness, but not Agreeableness, also tends to be associated with more physical activity.

A related way to think about physical activity is how often the individual spends doing sedentary activities, such as watching TV or reading a book. The relation between personality and such sedentary behaviors diverge somewhat from its relation with physical activity (Sutin et al., 2016). Although higher Neuroticism and lower Conscientiousness are associated with more time spent in sedentary behavior, Extraversion and Openness are primarily unrelated to sedentary behavior. Individuals who score higher in either Extraversion or
Openness tend to be involved in many different kinds of activity (Stephan, Boiché, Canada, \& Terracciano, 2013), some of which may involve being sedentary. As such, individuals who score higher in these traits are just as likely to engage in sedentary as non-sedentary behavior. For individuals high in Neuroticism and low in Conscientiousness, the dual behaviors of less physical activity and more sedentary behavior may contribute to higher BMI and greater risk of obesity over time.

Diet and food-related behaviors. Diet is a second behavior that is associated strongly with weight and weight gain. There are at least two food-related behaviors that are important for understanding obesity. The first behavior is what the individual actually eats. The second behavior is how the food is consumed, including overeating and meal rhythmicity. Personality traits are associated with both of these aspects of food-related behavior.

Somewhat surprisingly, Openness is the trait with the strongest and most consistent associations with the type of food that individuals consume. Individuals who score higher in the tendency to be open-minded and unconventional eat a healthier diet, including a Mediterranean diet (Mõttus et al., 2012, 2013). Individuals higher in Conscientiousness and Extraversion also eat healthier diets, whereas individuals higher in Neuroticism are more likely to eat convenience food than healthy food (Mõttus et al., 2012; Sutin \& Terracciano, 2016b).

Neuroticism and Conscientiousness are more strongly related to how individuals consume food than what food is consumed. Individuals high in Neuroticism, for example, are more likely to suffer from disordered eating, including eating too much 
and extreme dieting behavior, both of which increase risk of weight gain (Elfhag \& Morey, 2008; Keller \& Siegrist, 2015). Individuals high in Conscientiousness tend to have healthier eating patterns. In addition to better control over their food intake, these individuals tend to have more consistent meal rhythmicity, such as eating at regular times over the course of the day and at consistent times day to day (Sutin \& Terracciano, 2016b).

It is of note that Openness is primarily unrelated to BMI (Armon et al., 2013; Jokela, Hintsanen, et al., 2013; Sutin et al., 2011), despite consistent evidence that individuals who score higher on this trait engage in behaviors that promote healthier weight. Likewise, the high energy and physical activity associated with Extraversion does not translate reliably into thinner bodies. There are perhaps other factors that push against the protective effects of diet and physical activity associated with these traits.

Physiological dysregulation. A second pathway through which personality contributes to BMI may be through physiological dysregulation. A number of studies have linked personality to biomarkers that are also known to be associated with weight. For example, personality is associated with c-reactive protein (CRP), an inflammatory marker that is associated with subsequent weight gain (Duncan et al., 2000). A meta-analysis of the association between personality and inflammation found that Conscientiousness was associated consistently with lower levels of inflammation even after accounting for a number of confounding factors (Luchetti, Barkley, Stephan, Terracciano, \& Sutin, 2014). In contrast, Neuroticism is not associated consistently with CRP but does tend to be associated with higher average levels of cortisol, a stress hormone associated with weight; Conscientiousness tends to be unrelated to it (Nater, Hoppmann, \& Klumb, 2010). This pattern suggests that there may be different physiological pathways between personality and weight for Neuroticism and Conscientiousness.

Other physiological pathways, such as through adipose-derived hormones, may also contribute to the association between personality and body weight. These hormones arise from the gut and send signals to the brain to regulate appetite. Leptin, for example, is known as the "satiety hormone" because it is excreted to tell the brain to stop eating once the stomach is full. Similar to insulin resistance, individuals can become insensitive to leptin's signal, which impairs the individual's ability to regulate food intake. Conscientiousness is associated with this adiposederived hormone: Individuals who are more disorganized and undisciplined tend to have higher levels of leptin circulating in their blood (Sutin, Zonderman, Uda, et al., 2013). This association suggests that the reduced sensitivity to satiety may be one reason that individuals lower in Conscientiousness tend to overeat. Leptin further mediates the relation between Conscientiousness and elevated body weight, whereas it is not associated with the other four traits (Sutin, Zonderman, Uda, et al., 2013). Individuals low in Conscientiousness tend not to listen to external signals; the association with leptin suggests these individuals do not listen to their body's internal signals either.

Context. Personality does not exist in a vacuum. Individuals move in a social world and aspects of the environment are likely to interact with personality traits to contribute to body weight. There are many ways to define context: Poverty, urban/rural, and a hostile/discriminatory 
environment are examples of contextual factors that are likely to have an impact on personality and weight. More work is needed to determine how specific aspects of the environment interact with personality to predict weight and weight gain. To address this issue, we started with a broad definition of context: the cultural context in which the individual lives.

As a starting point, we examined how personality is associated with BMI in a sample of participants who were all of Mexican origin (Sutin, Rogers et al., 2015). This sample included participants born in Mexico and living in Mexico, participants born in Mexico and living in the United States ( $1^{\text {st }}$ generation immigrants), participants born and living in the United States to parents born in Mexico ( $2^{\text {nd }}$ generation immigrants), and participants born and living in the United States to parents of Mexican origin but who were also born in the United States ( $3^{\text {rd }}$ generation immigrants). With a sample that varied in terms of nativity, we could test whether the association between personality and BMI depended on the broader cultural context of the individual (i.e., living in the United States versus Mexico).

In the total sample, higher Neuroticism and lower Conscientiousness were both associated with higher BMI. The association with Neuroticism, however, was moderated by nativity. For participants born in the United States, there was a positive association between Neuroticism and body weight, whereas there was no association for participants in Mexico. Interestingly, for participants who were $1^{\text {st }}$ generation immigrants, this association was somewhere in between. Individuals who score higher in Neuroticism regulate their negative emotions in various ways. In the United States, it may be more socially acceptable to regulate nega- tive emotions with food, which increases risk of higher body weight. Nativity did not moderate the association between Conscientiousness and BMI, which indicated the protective association for Conscientiousness was pervasive across nativity.

A second way to address context is examine whether the association between personality and BMI varies across countries. If the association between personality and BMI is similar across countries that are culturally very different, it would suggest that the association between personality and BMI is not dependent on culture. If replicable differences emerge, however, it suggests that aspects of the environment may shape how personality is expressed and that the association with health outcomes, such as BMI and obesity, may be culturespecific. The majority of studies on personality and BMI have used samples of participants from Western countries. In samples from North America, Europe, and Australia, Conscientiousness is a powerful and consistent predictor of BMI (Jokela, Hintsanen, et al., 2013; Magee \& Heaven, 2011; Mõttus et al., 2013; Sutin \& Terracciano, 2016b). Of note, the typical association between Conscientiousness and BMI was found in a large sample from the island of Sardinia, Italy (Terracciano et al., 2009). This is noteworthy because compared to US samples, the Sardinian population has a relatively lower rate of obesity and has the healthier Mediterranean diet. The few studies with samples from Eastern countries that include a measure of Conscientiousness surprisingly find no association between this trait and BMI. In samples from South Korea (Shim et al., 2014) and China and Japan (Sutin, Stephan et al., 2015), Conscientiousness is unrelated to body weight. Given the overwhelming evidence for the protective role of Conscientiousness in Western samples, this 
unusual finding should be interpreted with caution until more samples are available to test for this association.

Interestingly, one effect was found in every sample of participants from Eastern countries: there was a positive association between Extraversion and BMI for men but not for women (Sutin et al., 2015). As reviewed above, this moderating effect of sex has also been found in some Western samples. The growing evidence that Extraversion is associated with greater BMI among men suggests that this association may be universal.

\section{Future Directions}

The present review of the literature summarized what is known about the relation between personality traits and BMI/obesity. There is still much to discover, however, about how personality contributes to weight gain across the lifespan. Future research needs to address a number of aspects about the nature of the relation between personality and weight. First, more attention is needed on the context in which personality is expressed. In addition to broad cultural context, more specific aspects of the cultural context (e.g., how personality interacts with public policy) could be addressed to identify specific contextual factors and how they shape the association between personality and health. Second, several physiological mechanisms that explain part of the association between personality and BMI have been identified, but there are likely to be others. Further, there are likely to be reciprocal relations between personality and physiological dysregulation. It is necessary to have multiple measures of both personality and biomarkers to address the longitudinal effect of dysregulation on personality development. Third, the trajectory of weight gain begins in childhood, and adolescent adiposity sets the foundation for adult weight (The, Suchindran, North, Popkin, \& Gordon-Larsen, 2010). It is thus critical to identify early life factors that contribute to this foundation. In the context of personality, there is evidence that the child's temperament is associated with his/her weight (Pulkki-Råback, Elovainio, Kivimäki, Raitakari, \& Keltikangas-Järvinen, 2005) and that personality measured in childhood predicts midlife BMI (Hampson et al., 2013). There may also be an intergenerational association between personality and child weight. That is, the personality of the parent may contribute to the child's weight. Parents who are more conscientious, for example, may have children with healthier body weight. To address this issue, more research needs to be done at the level of the family and include the personality and BMI of parents and their children.

The identification of replicable associations between personality and longevity sparked a line of research into how traits contribute to health across the lifespan. An individual's body weight is one factor in the sequence between personality and longevity. Although much work still needs to be done, there is substantial evidence for replicable associations between personality and BMI/ obesity and evidence that these associations play out over time, are mediated by both behavioral and physiological mechanisms, and may be modified by the context in which the individual lives.

\section{Acknowledgements}

ARS was supported by a grant from the Eunice Kennedy Shriver National Institute of Child Health and Human Development (1R15HD083947).

\section{References}

Armon, G., Melamed, S., Shirom, A., Shapira, I., \& 
Berliner, S. (2013). Personality traits and body weight measures: Concurrent and across-time associations. European Journal of Personality, 27, 398-408. doi: 10.1002/per.1902

Barrick, M. R., \& Mount, M. K. (1991). The Big 5 personality dimensions and job-performance: A metaanalysis. Personnel Psychology, 44, 1-26. doi: 10.1111/ j.1744-6570.1991.tb00688.x

Brummett, B. H., Babyak, M. A., Williams, R. B., Barefoot, J. C., Costa, P. T., \& Siegler, I. C. (2006). NEO personality domains and gender predict levels and trends in body mass index over 14 years during midlife. Journal of Research in Personality, 40, 222236. doi: 10.1016/j.jrp.2004.12.002

Chapman, B. P., Fiscella, K., Duberstein, P., Coletta, M., \& Kawachi, I. (2009). Can the influence of childhood socioeconomic status on men's and women's adult body mass be explained by adult socioeconomic status or personality? Findings from a national sample. Health Psychology, 28, 419-427. doi: 10.1037/a0015212

Chapman, B. P., Fiscella, K., Kawachi, I., \& Duberstein, P. R. (2010). Personality, socioeconomic status, and all-cause mortality in the United States. American Journal of Epidemiology, 171, 83-92. doi: 10.1093/aje/ kwp323

Duncan, B. B., Schmidt, M. I., Chambless, L. E., Folsom, A. R., Carpenter, M., \& Heiss, G. (2000). Fibrinogen, other putative markers of inflammation, and weight gain in middle-aged adults: The ARIC study. Atherosclerosis Risk in Communities. Obesity Research, 8, 279-286. doi: 10.1038/oby.2000.33

Elfhag, K., \& Morey, L. C. (2008). Personality traits and eating behavior in the obese: Poor self-control in emotional and external eating but personality assets in restrained eating. Eating Behavior, 9, 285-293. doi: 10.1016/j.eatbeh.2007.10.003

Ferguson, C. J., Muñoz, M. E., \& Winegard, B. (2012). The influence of heritability, neuroticism, maternal warmth and media use on disordered eating behaviors: A prospective analysis of twins. Psychiatrity Quarterly, 83, 353-360. doi: 10.1007/s11126-012-9205-7

Finucane, M. M., Stevens, G. A., Cowan, M. J., Danaei, G., Lin, J. K., Paciorek, C. J., et al. (2011). National, regional, and global trends in body-mass index since 1980: Systematic analysis of health examination surveys and epidemiological studies with 960 country-years and $9 \cdot 1$ million participants. Lancet, 377, 557-567. doi: 10.1016/S0140-6736(10)62037-5

Friedman, H. S., Kern, M. L., Hampson, S. E., \& Duckworth, A. L. (2014). A new life-span approach to conscientiousness and health: Combining the pieces of the causal puzzle. Developmental Psychology, 50, 1377-1389. doi: 10.1037/a0030373

Friedman, H. S., Tucker, J. S., Tomlinson-Keasey, C., Schwartz, J. E., Wingard, D. L., \& Criqui, M. H. (1993). Does childhood personality predict longevity? Journal of Personality and Social Psychology, 65, 176-185. doi: 10.1037/0022-3514.65.1.176

Gortmaker, S. L., Swinburn, B. A., Levy, D., Carter, R., Mabry, P. L., Finegood, D. T., ... Moodie, M. L. (2011). Changing the future of obesity: Science, policy, and action. Lancet, 378, 838-847. doi: 10.1016/S0140-6736 (11)60815-5

Hampson, S. E., Edmonds, G. W., Goldberg, L. R., Dubanoski, J. P., \& Hillier, T. A. (2013). Childhood conscientiousness relates to objectively measured adult physical health four decades later. Health Psychology, 32, 925-928. doi: 10.1037/a0031655

Jokela, M., Batty, G. D., Nyberg, S. T., Virtanen, M., Nabi, H., Singh-Manoux, A., \& Kivimäki, M. (2013). Personality and all-cause mortality: individualparticipant meta-analysis of 3,947 deaths in 76,150 adults. American Journal of Epidemiology, 178, 667675. doi: 10.1093/aje/kwt170

Jokela, M., Hintsanen, M., Hakulinen, C., Batty, G. D., Nabi, H., Singh-Manoux, A., \& Kivimäki, M. (2013). Association of personality with the development and persistence of obesity: A meta-analysis based on individual-participant data. Obesity Reviews, 14, 315323. doi: 10.1111/obr.12007

Keller, C., \& Siegrist, M. (2015). Does personality influence eating styles and food choices? Direct and indirect effects. Appetite, 84, 128-138. doi: 10.1016/j. appet.2014.10.003

Kendler, K. S., \& Myers, J. (2010). The genetic and environmental relationship between major depression and the five-factor model of personality. Psychological Medicine, 40, 801-806. doi: 10.1017/S003329170999 1140

Kern, M. L., \& Friedman, H. S. (2008). Do conscientious individuals live longer? A quantitative review. Health Psychology, 27, 505-512. doi: 10.1037/0278-6133.27.5. 
505

Luchetti, M., Barkley, J. M., Stephan, Y., Terracciano, A., \& Sutin, A. R. (2014). Five-factor model personality traits and inflammatory markers: New data and a metaanalysis. Psychoneuroendocrinology, 50, 181-193. doi: 10.1016/j.psyneuen.2014.08.014

Magee, C. A., \& Heaven, P. C. L. (2011). Big-Five personality factors, obesity and 2-year weight gain in Australian adults. Journal of Research in Personality, 45, 332-335. doi: 10.1016/j.jrp.2011.02.009

McCrae, R. R., \& Costa, P. T. (2003). Personality in adulthood: A Five-Factor Theory perspective (2nd ed.). New York: Guilford Press.

McCrae, R. R., Costa P. T., Jr., Martin, T. A., Oryol, V. E., Rukavishnikov, A. A., Senin, I. G., ... Urbánek, T. (2004). Consensual validation of personality traits across cultures. Journal of Research in Personality, 38, 179-201. doi: 10.1016/S0092-6566(03)00056-4

McCrae, R. R., Terracciano, A., et al. (2005). Universal features of personality traits from the observer's perspective: Data from 50 cultures. Journal of Personality and Social Psychology, 88, 547-561. doi: 10.1037/0022-3514.88.3.547

Mõttus, R., McNeill, G., Jia, X., Craig, L. C., Starr, J. M., \& Deary, I. J. (2013). The associations between personality, diet and body mass index in older people. Health Psychology, 32, 353-360. doi: 10.1037/a0025537 Mõttus, R., Realo, A., Allik, J., Deary, I. J., Esko, T., \& Metspalu, A. (2012). Personality traits and eating habits in a large sample of Estonians. Health Psychology, 31, 806-814. doi:10.1037/a0027041

Nater, U. M., Hoppmann, C., \& Klumb, P. L. (2010). Neuroticism and conscientiousness are associated with cortisol diurnal profiles in adults: Role of positive and negative affect. Psychoneuroendocrinology, 35, 1573-1577. doi: 10.1016/j.psyneuen.2010.02.017

Noftle, E. E., \& Robins, R. W. (2007). Personality predictors of academic outcomes: Big Five correlates of GPA and SAT Scores. Journal of Personality and Social Psychology, 93, 116-130. doi: 10.1037/0022-3514. 93.1.116

Ogden, C. L., Yanovski, S. Z., Carroll, M. D., \& Flegal, K. M. (2007). The epidemiology of obesity. Gastroenterology, 132, 2087-2102. doi: 10.1053/j. gastro.2007.03.052

Pulkki-Råback, L., Elovainio, M., Kivimäki, M., Raitakari,
O. T., \& Keltikangas-Järvinen, L. (2005). Temperament in childhood predicts body mass in adulthood: The Cardiovascular Risk in Young Finns Study. Health Psychology, 24, 307-315. doi: 10.1037/0278-6133.24.3. 307

Rhodes, R. E., \& Smith, N. E. I. (2006). Personality correlates of physical activity: A review and metaanalysis. British Journal of Sports Medicine, 40, 958-965. doi: 10.1136/bjsm.2006.028860

Roberts, B. W., \& DelVecchio, W. F. (2000). The rankorder consistency of personality traits from childhood to old age: A quantitative review of longitudinal studies. Psychological Bulletin, 126, 3-25. doi: 10.1037/00332909.126.1.3

Shim, U., Kim, H. N., Roh, S. J., Cho, N. H., Shin, C., Ryu, S., ... Kim, H. L. (2014). Personality traits and body mass index in a korean population. PLoS ONE, 9, e90516. doi: 10.1371/journal.pone.0090516

Stephan, Y., Boiché, J., Canada, B., \& Terracciano, A. (2013). Association of personality with physical, social, and mental activities across the lifespan: Findings from US and French samples. British Journal of Psychology. doi: 10.1111/bjop.12056

Sutin, A. R., Costa, P. T., Chan, W., Milaneschi, Y., Eaton, W. W., Zonderman, A. B., ... Terracciano, A. (2013). I know not to, but I can’t help it: Weight gain and changes in impulsivity-related personality traits. Psychological Science. doi:10.1177/0956797612469212

Sutin, A. R., Ferrucci, L., Zonderman, A. B., \& Terracciano, A. (2011). Personality and obesity across the adult life span. Journal of Personality and Social Psychology, 101, 579. doi: 10.1037/a0024286

Sutin, A. R., Rogers, D. L., Mercado, A., Weimer, A., Rodriguez, C. C., Gonzalez, M., ... Terracciano, A. (2015). The association between personality traits and body mass index varies with nativity among individuals of Mexican origin. Appetite, 90, 74-79. doi:10.1016/j.appet.2015.02.036

Sutin, A. R., Stephan, Y., Luchetti, M., Artese, A., Oshio, A., \& Terracciano, A. (2016). The five factor model of personality and physical inactivity: A meta-analysis of 16 samples. Journal of Research in Personality, 63, 2228. doi: 10.1016/j.jrp.2016.05.001

Sutin, A. R., Stephan, Y., Wang, L., Gao, S., Wang, P., \& Terracciano, A. (2015). Personality traits and body mass index in Asian populations. Journal of Research 
in Personality, 58, 137-142. doi: 10.1016/j.jrp.2015.07. 006

Sutin, A. R., \& Terracciano, A. (2016a). Five factor model personality traits and the objective and subjective experience of body weight. Journal of Personality, 84, 102-112. doi: 10.1111/jopy.12143

Sutin, A. R., \& Terracciano, A. (2016b). Personality traits and body mass index: Modifiers and mechanisms. Psychology and Health, 31, 259-275. doi: 10.1080/ 08870446.2015.1082561

Sutin, A. R., \& Zonderman, A. (2012). Depressive symptoms are associated with weight gain among women. Psychological Medicine, 42, 2351-2360. doi: 10.1017/S0033291712000566

Sutin, A. R., Zonderman, A. B., Ferrucci, L., \& Terracciano, A. (2013). Personality traits and chronic disease: Implications for adult personality development. Journals of Gerontology Series B Psychological Science and Social Sciences. doi: 10.1093/geronb/gbt036

Sutin, A. R., Zonderman, A. B., Uda, M., Deiana, B., Taub, D. D., Longo, D. L., ... Terracciano, A. (2013). Personality traits and leptin. Psychosomatic Medicine, 75, 505-509. doi: 10.1097/PSY.0b013e3182919ff4

Terracciano, A., Löckenhoff, C. E., Zonderman, A. B.,
Ferrucci, L., \& Costa, P. T. (2008). Personality predictors of longevity: Activity, emotional stability, and conscientiousness. Psychosomatic Medicine, 70, 621627. doi: 10.1097/PSY.0b013e31817b9371

Terracciano, A., Sutin, A. R., McCrae, R. R., Deiana, B., Ferrucci, L., Schlessinger, D., ... Costa, P. T. (2009). Facets of personality linked to underweight and overweight. Psychosomatic Medicine, 71, 682-689. doi: 10.1097/PSY.0b013e3181a2925b

The, N. S., Suchindran, C., North, K. E., Popkin, B. M., \& Gordon-Larsen, P. (2010). Association of adolescent obesity with risk of severe obesity in adulthood. JAMA-Journal of the American Medical Association, 304, 2042-2047. doi: 10.1001/jama.2010.1635

Turiano, N. A., Chapman, B. P., Gruenewald, T. L., \& Mroczek, D. K. (2015). Personality and the leading behavioral contributors of mortality. Health Psychology, 34, 51-60. doi: $10.1037 /$ hea0000038

Wilson, K. E., \& Dishman, R. K. (2015). Personality and physical activity: A systematic review and metaanalysis. Personality and Individual Differences, 27, 230-242. doi: 10.1016/j.paid.2014.08.023

- 2016.11.16受稿, 2017.1.4受理一 